http://jmscr.igmpublication.org/home/ ISSN (e)-2347-176x ISSN (p) 2455-0450 crossref DOI: https://dx.doi.org/10.18535/jmscr/v8i4.91

\title{
Use of Plasmapheresis in hypertriglyceridemia-induced acute recurrent pancreatitis: a challenging experience
}

\author{
Authors \\ Hussain A. Jahlan ${ }^{1 *}$, Esraa M. Hasan ${ }^{2}$, Hanan H. Almalki ${ }^{3}$, \\ Eman A. Almhjani ${ }^{4}$, Wejdan A. Mobasher ${ }^{5}$ \\ ${ }^{1-5}$ Endocrinology Unit, Department of Medicine, Aseer Central Hospital, Abha, Saudi Arabia \\ *Corresponding Author \\ Dr Hussain A. Jahlan \\ Endocrinology Unit, Department of Medicine, Aseer Central Hospital, Abha, Saudi Arabia
}

\begin{abstract}
Background: Hypertriglyceridemia is a well-established but underestimated cause of acute and recurrent pancreatitis. Initial management of patients with Hypertriglyceridemia-induced pancreatitis includes treatment of acute pancreatitis and reduction of serum triglyceride levels to $<500 \mathrm{mg} / \mathrm{dL}$ by administering intravenous insulin and plasmapheresis in selected patients with severe HTGP. Patients with HTGP require long-term therapy consists of both pharmacologic therapy and dietary fat restriction.

Aim: To affirm the use of plasmapheresis in treatment and prevention of acute recurrent hypertriglyceridemic pancreatitis.

Case: A 35-year-old female with familial hypertriglyceridemia resistant to medical therapy and a history of recurrent attacks of hypertriglyceridemic pancreatitis presented with acute pancreatitis and TG level of 1414 mg $\backslash d l$. The patient was admitted and underwent repeated sessions of plasmapheresis along with medical therapy which resulted in a significant reduction of $T G$ and a complete resolution of her pancreatitis symptoms.
\end{abstract}

Keywords: Hypertriglyceridemia, Hyperlipidemia, Acute pancreatitis, Plasmapheresis.

\section{Background}

Acute pancreatitis is a common condition with different causes; gall stones and alcohol are the most common $^{(1)}$. Hyperlipidemia including hypertriglyceridemia is less frequent cause, but it is one of the well-known underlying causes of acute pancreatitis in $7 \%$ of the cases being the most frequent after gall stones and alcohol ${ }^{(2)}$.

Pancreatitis secondary to HTG, presents as an episode of acute pancreatitis or recurrent $\mathrm{AP}^{(3)}$. HTG occurs as a result of multiple genetic mutations in $5 \%$ of the cases and can be secondary to other causes (diabetes, obesity, pregnancy, alcohol or drugs) $)^{(4)}$.

\section{Case Report}

A thirty-five years old female patient with a history of hypothyroidism and hypertension for five years on treatment, attended to the emergency room complaining of epigastric abdominal pain and vomiting for one day. The pain was severe, progressive and radiating to the back without reliving or aggravating factors. It was associated with nausea and vomiting for five times. There was 
no diarrhea, constitutional symptoms, chest pain, chronic heartburn or NSAIDs use. The patient denied any history of invasive procedures or biliary colic. She had three similar presentations with ICU admission. Besides, the patient had a history of recurrent renal stones for the last three years. Never smokes neither drinks alcohol. She was working as a nurse, living with her husband and known to have primary infertility for six years without a clear cause. Further questioning revealed that the patient was diagnosed with hypertriglyceridemia a year ago but was non adherent and stopped the treatment on the second hospitalization. Regarding family history, her mother developed similar complaint in the past with no confirmed diagnosis. Upon examining her, she was conscious, alert, oriented and looking sick but vital signs were normal with a BMI of 26.8. Abdominal examination showed soft abdomen with mild tenderness in epigastric area and liver span of $15 \mathrm{~cm}$. Chest, cardiovascular and neurological examination were completely normal with no skin pigmentation or lesions noticed.

Differential diagnosis initially includes acute pancreatitis, perforated ulcer, cholecystitis, esophagitis, gastritis, peptic ulcer, myocardial infarction, pericarditis and small bowel obstruction.

Laboratory investigations revealed normal CBC, LFT, coagulation profile, renal functions, electrolyte and TFT apart of WBCs which were 14.000 cell/microliter, albumin of $2.5 \mathrm{~g} / \mathrm{dl}$ and serum sodium of $129 \mathrm{mmol} / \mathrm{L}$. Lactate level in serum was $32 \mathrm{mg} / \mathrm{dl}$. High Lipase and amylase levels (750u/1 and $350 \mathrm{u} / \mathrm{l}$ respectively) were found. All serology of $\mathrm{HBV}, \mathrm{HCV}$ and HIV came negative. Lipid profile showed that total cholesterol level was $327 \mathrm{mg} / \mathrm{dl}$ (normal range $=50-200 \mathrm{mg} / \mathrm{dl}$ ), HDL cholesterol was $19 \mathrm{mg} / \mathrm{dl}$ (normal $=30-70 \mathrm{mg} / \mathrm{dl}$ ), and triglyceride was $1414 \mathrm{mg} / \mathrm{dl}$ (normal $=50-200 \mathrm{mg} / \mathrm{dl}$ ). Moderate peripancreatic fat stranding with minimal rim of fluid, liver size up to $27 \mathrm{~cm}$ in craniocaudal dimension, and parenchymal density of $10 \mathrm{HU}$ observed in abdominal CT which highly suggest pancreatitis and fatty liver(figure1).

Final diagnosis conclusion was hypertriglyceridemia induced recurrent pancreatitis.

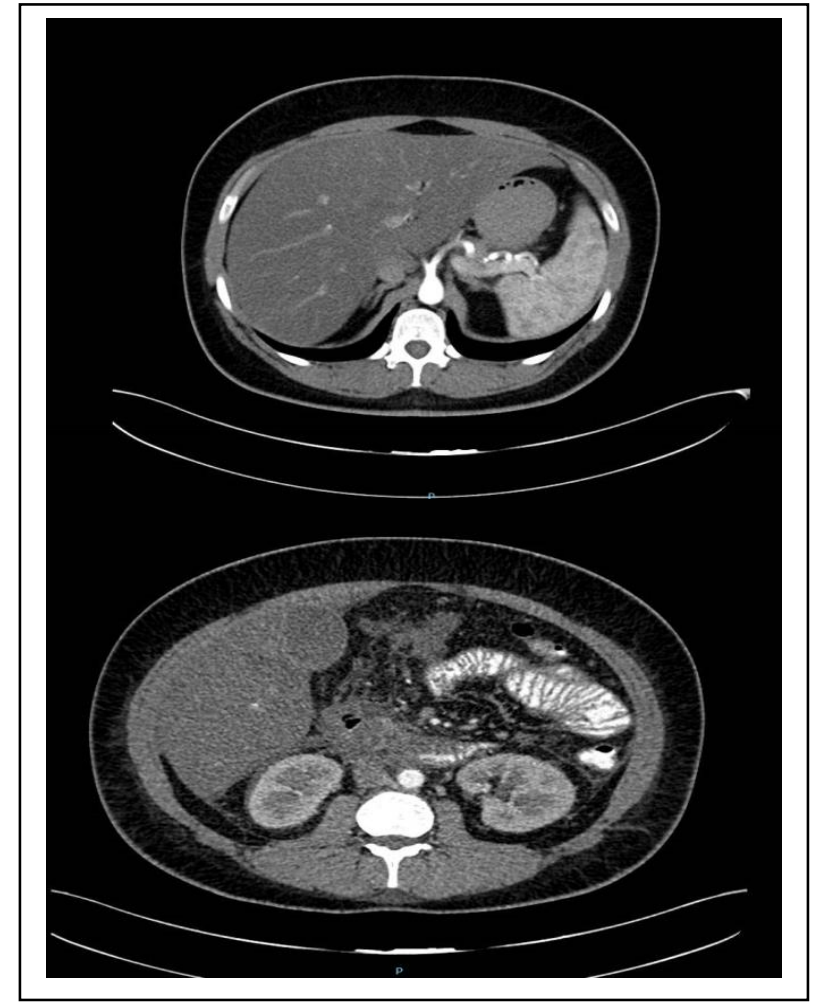

Figure 1: Abdominal CT showing evidence of pancreatitis and fatty liver

Patient was admitted, received two litres of IVF and kept NPO, on high rate maintenance fluid, analgesia, insulin infusion, omega3 and fenofibrate. She deteriorated and then transferred to ICU. Plasmapheresis started and repeated three times (U.F $3.0 \mathrm{~kg}, 7$ bottles of NS each contains $300 \mathrm{ml} \mathrm{NS}$ $+100 \mathrm{ml}$ human albumin20\% + calcium gluconate) . The patient dramatically improved and was discharged on rosuvastatin, fenofibrate and omega3 along with her thyroxin, omeprazole and amlodipine (Figure 2).

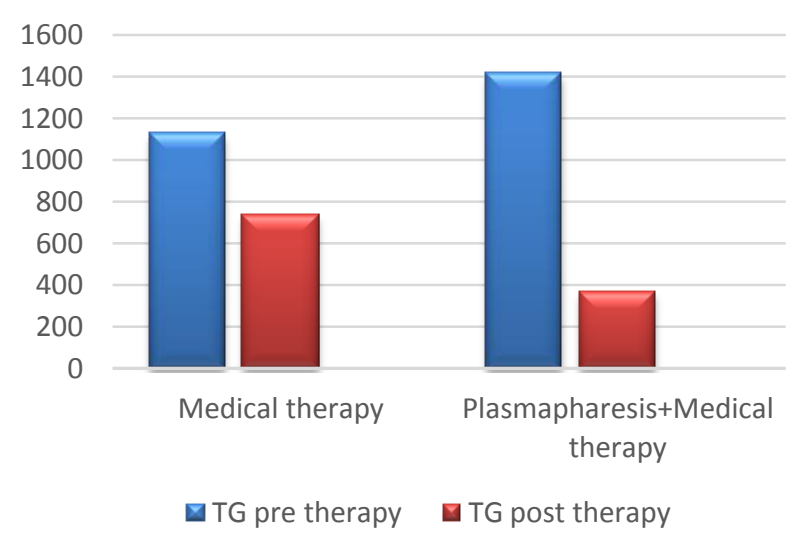

Figure 2: Reduction in TG levels with different treatment modalities 


\section{Discussion}

Familial Hypertriglyceridemia is an autosomal dominant disorder characterized by moderate increase in the serum triglyceride concentration (200 to $500 \mathrm{mg} / \mathrm{dl}[2.3$ to $5.6 \mathrm{mmol} / \mathrm{L}]$ ). Hypertriglyceridemia is defined as fasting serum triglyceride level of exceeding $150 \mathrm{mg} / \mathrm{dl}$ (1.7 $\mathrm{mmol} / \mathrm{L}$ ) and classified into mild (150 to $199 \mathrm{mg} / \mathrm{dl}$ 1.7 to $2.2 \mathrm{mmol} / \mathrm{L}$ ), moderate (200 to $999 \mathrm{mg} / \mathrm{dl}, 2.3$ to $11.2 \mathrm{mmol} / \mathrm{L}$ ), and severe (1000 to $1999 \mathrm{mg} / \mathrm{dl}$, 11.3 to $22.5 \mathrm{mmol} / \mathrm{L})$. Very severe $(\geq 2000$ $\mathrm{mg} / \mathrm{dl},>22.6 \mathrm{mmol} / \mathrm{L}$ ) levels more than $1000 \mathrm{mg} / \mathrm{dl}$ $(11.3 \mathrm{mmol} / \mathrm{L})$ considered a significant risk factor for pancreatitis ${ }^{(5)}$. The risk of having acute pancreatitis is nearly $5 \%$ when serum triglycerides exceed $1000 \mathrm{mg} / \mathrm{dl}(11.3 \mathrm{mmol} / \mathrm{L})$ and may raise up to $10 \%$ to $20 \%$ with triglycerides over $2000 \mathrm{mg} / \mathrm{dl}$ (22.6 mmol/L) ${ }^{(6)}$.Types I (high chylomicrons), IV (high very low-density lipoprotein [VLDL]), and V (high chylomicrons and VLDL) dyslipidemias (Fredrickson's classification) are associated with higher risk of acute pancreatitis. Type I dyslipidemia can cause acute pancreatitis in absence of exacerbating condition unlike type IV and $\mathrm{V}$ dyslipidemia which need environmental or hormonal factors ${ }^{(7)}$. The level of triglyceride is associated with the degree of acute pancreatitis. Other factors including the severity of the underlying pancreatic injury, pancreatic lipase activity and the efficiency of clearing free fatty acid from the serum influence the severity of acute pancreatitis $^{(8)}$. The exact mechanisms in hypertriglyceridemia-induced pancreatitis are unclear. Hydrolysis of triglycerides by pancreatic lipase with the local release toxic free fatty acids (FFAs) has been described as the pathogenetic mechanism. Hyperviscosity from excessive TGs might minimize circulatory flow in pancreatic capillaries leading to ischemia and further damage of pancreatic acinar cells ${ }^{(9)}$. HTGP clinically manifests as other causes of acute pancreatitis but tends to cause severe pancreatitis with persistent organ failure compared to other causes of pancreatitis $^{(10)}$. Eruptive xanthomas, hepatosplenomegaly and lipemia retinalis (if TG levels exceed $4000 \mathrm{mg} / \mathrm{dL}$ ) are the clinical signs that may observed in $\mathrm{HTGP}^{(11)}$. Pseudo-hyponatremia and normal amylase can be caused by high triglyceride levels which require serial dilutions to provide adequate results ${ }^{(12)}$. Multiple small studies on HTGP management have evaluated the use of insulin, heparin, or both. Many series have also reported use of plasmapheresis to reduce TG levels. In a series of seven patients with severe hypertriglyceridemia intravenous infusion of insulin at a rate of 0.05-2 U/kg/day was used. After Two and a half days the serum triglyceride level remained lower than $400 \mathrm{mg} / \mathrm{dL}$. There were no complications during the treatment. The long treatment included basal insulin (for diabetic and non-diabetic patients) and fibrates ${ }^{(13)}$.In another report, 5 patients were diagnosed with HTGP, treated with I.V heparin and I.V insulin decreased triglyceride levels to less than $10 \mathrm{mmol} / \mathrm{l}$ within 2.8 days, the amylase and lipase levels returned to normal after 3-4 days ${ }^{(14)}$. According to the American Society for Apheresis, hypertriglyceridemia is a class III indication for plasma exchange ${ }^{(15)}$. Plasmapheresis reduced serum triglycerides faster than conservative treatment is the conclusion obtained from observational cohort study for 103 patients with 111 episodes of HTGP treated with PE. The mean reduction in triglycerides during PE was twice the reduction observed during conservative treatment representing $59 \%{ }^{(16)}$. One PE procedure is possibly decreasing the length of hospital stay in patients admitted with HTP, while regular plasmapheresis decreases the incidence of pancreatitis and may be useful for preventing acute pancreatitis ${ }^{(17,18)}$. In a case series of 10 patients were admitted to the intensive care unit with a diagnosis of acute severe hyperlipidemic pancreatitis. They underwent standard treatment, followed by plasmapheresis within 48 hours of admission. Kyriakidis AV et al, 2006 concluded that plasmapheresis lowered the triglyceride and lipid levels in all cases in addition to standard treatment $^{(19)}$. Joglekar $\mathrm{K}$ eta al, 2017 conducted a research to analyse the outcomes of patients treated with plasmapheresis for severe HTG-associated 
pancreatitis. The researchers reported that the average TG level before plasmapheresis was 3532 $\mathrm{mg} / \mathrm{dl}$ (range: $2524-4562 \mathrm{mg} / \mathrm{dl} ; 39.9 \mathrm{mmol} / \mathrm{l}$; range: 28.5-51.6 mmol/l). All patients were completely recovered, with a remarkable improvement in TG levels after plasmapheresis. The mean number of sessions was 1.3 (range 1-2), and mean TG level after plasmapheresis was $1051 \mathrm{mg} / \mathrm{dl}$ (range: 509$1771 \mathrm{mg} / \mathrm{dl}$; $11.9 \mathrm{mmol} / \mathrm{l}$; range: $5.8-20 \mathrm{mmol} / \mathrm{l}$ ). After the first session, TG showed an average reduction in its level of $2481 \mathrm{mg} / \mathrm{dl}$ (range 753$3750 \mathrm{mg} / \mathrm{dl}$; $28 \mathrm{mmol} / \mathrm{l}$; range: $8.5-42.4 \mathrm{mmol} / \mathrm{l}$ ) or approximately $70 \%$. None of the patients developed complications related to plasmapheresis ${ }^{(20)}$.In a large randomized trial, the use of statin therapy was associated with a lower risk of pancreatitis although Previous case reports and pharmacoepidemiologic studies have demonstrated an association between statin therapy and increased risk of pancreatitis ${ }^{(21)}$.

The patient showed a significant response for the given medications based on the standard protocol besides adjusting dietary habits. Recurrence was recorded twice when she stopped treatment and was non adherent to physicians' advices.

\section{Conclusion}

This report emphasizes the effect of the plasmapheresis in the treatment and prevention of HTGP. However, large multicenter studies are needed to optimize future management guidelines for patients with HTGP.

\section{Conflict of interest: Nil}

\section{References}

1. Toskes PP. Hyperlipidemic pancreatitis. Gastroenterol Clin North Am. 1990;19:78391.

2. Seales CE, Ooi TC. Underrecognition of chylomicronemia as a cause of acute pancreatitis. CMAJ. 1992;147:1806-8.

3. Yadav, Dhiraj, and C. S. Pitchumoni. "Issues in hyperlipidemic pancreatitis." Journal of clinical gastroenterology 36.1 (2003): 54-62.
4. Hegele RA. Monogenic dyslipidemias: Window on determinants of plasma lipoprotein metabolism. Am J Hum Genet. 2001;69:1161-77.

5. Berglund L, Brunzell JD, Goldberg AC, Goldberg IJ, Sacks F, Murad MH, Stalenhoef AF. Evaluation and treatment of hypertriglyceridemia: an Endocrine Society clinical practice guideline. The Journal of Clinical Endocrinology \& Metabolism. 2012 Sep 1; 97(9):2969-89.

6. Scherer J, Singh V, Pitchumoni CS, Yadav D. Issues in hypertriglyceridemic pancreatitis-an update. Journal of clinical gastroenterology. 2014 Mar; 48(3):195.

7. Fredrickson DS. An international classification of hyperlipidemias and hyperlipoproteinemias. Annals of internal medicine. 1971 Sep 1; 75(3):471-2.

8. Linares CL, Pelletier AL, Czernichow S, Vergnaud AC, Bonnefont-Rousselot D, Levy P, Ruszniewski P, Bruckert E. Acute pancreatitis in a cohort of 129 patients referred for severe hypertriglyceridemia. Pancreas. 2008 Jul 1; 37(1):13-2.

9. Saharia P, Margolis S, Zuidema GD, Cameron JL. Acute pancreatitis with hyperlipemia: studies with an isolated perfused canine pancreas. Surgery. 1977;82:60-67.

10. Nawaz, Haq, et al. Elevated serum triglycerides are independently associated with persistent organ failure in acute pancreatitis. American Journal of Gastroenterology 110.10 (2015): 1497-1503.

11. Nayak, Keshav R., and Richard G. Daly. "Eruptive xanthomas associated with hypertriglyceridemia and new-onset diabetes mellitus." New England Journal of Medicine 350.12 (2004): 1235-1235.

12. Howard JM, Reed J. Pseudohyponatremia in acute hyperlipemic pancreatitis. A potential pitfall in therapy. Arch Surg 1985; 120:1053. 
13. Tamez-Pérez HE, Sáenz-Gallegos R, Hernández-Rodríguez $\mathrm{K}$, et al. [Insulin therapy in patients with severe hypertriglyceridemia]. Rev Med Inst Mex Seguro Soc 2006; 44:235.

14. Henzen C, Röck M, Schnieper C, Heer K. [Heparin and insulin in the treatment of acute hypertriglyceridemia-induced pancreatitis]. Schweiz Med Wochenschr 1999; 129:1242.

15. Connelly-Smith L, Dunbar NM. The 2019 guidelines from the American Society for Apheresis: what's new? CurrOpinHematol 2019; 26:461.

16. Gubensek J, Buturovic-Ponikvar J, Romozi K, Ponikvar R. Factors affecting outcome in acute hypertriglyceridemic pancreatitis treated with plasma exchange: an observational cohort study. PLoS One 2014; 9:e102748.

17. Gavva C, Sarode R, Agrawal D, Burner J. Therapeutic plasma exchange for hypertriglyceridemia induced pancreatitis: A rapid and practical approach. TransfusApher Sci 2016; 54:99.

18. Piolot A, Nadler F, Cavallero E, et al. Prevention of recurrent acute pancreatitis in patients with severe hypertriglyceridemia: value of regular plasmapheresis. Pancreas 1996; 13:96.

19. Kyriakidis AV, Raitsiou B, Sakagianni A, Harisopoulou V, Pyrgioti M, et al. (2006) Management of acute severe hyperlipidemic pancreatitis. Digestion 73: 259-64.

20. Joglekar K, Brannick B, Kadaria D, Sodhi A. Therapeutic plasmapheresis for hypertriglyceridemia-associated acute pancreatitis: case series and review of the literature. Therapeutic advances in endocrinology and metabolism. 2017 Apr; 8(4):59-65.

21. Preiss D, Tikkanen MJ, Welsh $\mathrm{P}$, et al. Lipid-modifying therapies and risk of pancreatitis: a meta-analysis. JAMA 2012; 308:804. 